\title{
ANNALES
}

UNIVERSITATIS MARIAE CURIE-SKŁODOWSKA

LUBLIN - POLONIA

VOL. LXV, NO. 2, 2011

SECTIO A

$191-202$

\author{
MAGDALENA SOBCZAK-KNEĆ, VIKTOR V. STARKOV \\ and JAN SZYNAL
}

\section{Old and new order of linear invariant family of harmonic mappings and the bound for Jacobian}

\author{
Dedicated to the memory of Professor Jan G. Krzyż
}

\begin{abstract}
The relation between the Jacobian and the orders of a linear invariant family of locally univalent harmonic mapping in the plane is studied. The new order (called the strong order) of a linear invariant family is defined and the relations between order and strong order are established.
\end{abstract}

1. A harmonic mapping $f$ in the unit disk $\mathbb{D}=\{z:|z|<1\}$ has a representation:

$$
f(z)=h(z)+\overline{g(z)}
$$

where $h$ and $g$ are holomorphic functions in $\mathbb{D}$.

We assume that $f$ is locally univalent and sense-preserving in $\mathbb{D}$, which is equivalent to $J_{f}(z)>0, z \in \mathbb{D}$, where $J_{f}(z)$ denotes the Jacobian of $f$ :

$$
J_{f}(z)=\left|h^{\prime}(z)\right|^{2}-\left|g^{\prime}(z)\right|^{2} .
$$

For the properties of harmonic mappings we can refer to surveys [1] and [2]. The notion of an affine and linear invariant family of univalent harmonic functions was proposed by Sheil-Small [6], and extended to local univalent mappings and then used efficiently by Schaubroeck in [5].

2000 Mathematics Subject Classification. Primary 30C55, Secondary 31A05.

Key words and phrases. Planar harmonic mappings, order of a linear invariant family, Jacobian. 
For any holomorphic automorphism $\varphi$ of $\mathbb{D}(\varphi \in \operatorname{Aut}(\mathbb{D}))$ we denote

$$
\begin{gathered}
T_{\varphi}(f(z))=\frac{f(\varphi(z))-f(\varphi(0))}{\varphi^{\prime}(0) h^{\prime}(\varphi(0))} \\
A_{\varepsilon}(f(z))=\frac{f(z)+\varepsilon \overline{f(z)}}{1+\varepsilon g^{\prime}(0)}, \quad|\varepsilon|<1, \quad \varepsilon \in \mathbb{C} .
\end{gathered}
$$

The transformations (1.3) and (1.4) are called the Koebe transform and the affine transform of a locally univalent harmonic function $f=h+\bar{g}$.

Put

$$
S_{\varphi, \varepsilon}(f(z))=A_{\varepsilon} \circ T_{\varphi}(f(z))
$$

In what follows $L$ denotes a family of locally univalent and sense-preserving harmonic functions $f=h+\bar{g}$ in $\mathbb{D}$ which have the expansion:

$$
f(z)=\sum_{n=2}^{\infty} a_{n}(f) z^{n}+z+\sum_{n=1}^{\infty} a_{-n}(f) \bar{z}^{n}, \quad z \in \mathbb{D} .
$$

A family $L$ is called an affine and linear invariant $A L I F$ if for any $f \in L$ the function $T_{\varphi}(f)$ and $A_{\varepsilon}(f)$ belong to $L$ for all $\varphi \in \operatorname{Aut}(\mathbb{D})$ and all $|\varepsilon|<1$.

A family $L$ is called $L I F$ (linear invariant family) if for any $f \in L$ and all $\varphi \in \operatorname{Aut}(\mathbb{D})$ the function $T_{\varphi}(f) \in L$.

The order of the family $L$ is defined as ord $L=\sup \left\{\left|a_{2}(f)\right|: f \in L\right\}$ (see [5] and [6]).

Example. The best known $A L I F$ family is the class $S_{H}$ of univalent harmonic mappings in $\mathbb{D}$ preserving orientation, as well as the subclasses $K_{H}$ of convex and $C_{H}$ of close-to-convex mapping [2].

A simple example of a family which is $L I F$ but not $A L I F$ is the family of locally univalent holomorphic functions in $\mathbb{D}$.

The properties of the transformation (1.3)-(1.5) have been used in [5] to obtain some bounds for the Jacobian $J_{f}(z)$ in terms of the order of a linear invariant family.

In this paper we give an improvement of one result from [5] (Theorem 2.1) and establish the relations between ord $L$ and the new order called the strong order $\overline{\operatorname{ord}} L$ defined below. Introduction of the new order $\overline{\operatorname{ord}} L$, allow us to prove Theorem 3.1 for arbitrary family $L$ which is an extension of Theorem 2.1, while $L$ is $A L I F$ family.

These relations depend on the upper bound for the Jacobian $J_{f}(z)$ in the terms of ord $L$ and $\overline{\operatorname{ord}} L$.

We end this introduction with two definitions and one lemma.

Definition 1.1. The affine hull of the family $L$ is defined as the set of functions

$$
A(L)=\left\{A_{\varepsilon}(f): f \in L,|\varepsilon|<1\right\} .
$$


Definition 1.2. The linear-affine hull of the family $L$ is defined as the set of functions

$$
\mathscr{L} A(L)=\left\{S_{\varphi, \varepsilon}(f): f \in L, \varphi \in \operatorname{Aut}(\mathbb{D}),|\varepsilon|<1\right\} .
$$

Lemma 1.1. If $L$ is a linear invariant family of harmonic functions $(L I F)$, then $A(L)$ is affine and linearly invariant $(A L I F)$.

Proof. By the definition of $A(L)$ it is enough to prove that $A(L)$ is a $(L I F)$. Every member of $A(L)$ has the form

$$
f_{\varepsilon}(z)=\frac{f+\varepsilon \bar{f}}{1+\varepsilon g^{\prime}(0)}=h_{\varepsilon}+\overline{g_{\varepsilon}}, \quad \varepsilon \in \mathbb{C}, \quad|\varepsilon|<1,
$$

where $f(z)=h(z)+\overline{g(z)} \in L$. The functions $h, g, h_{\varepsilon}$ and $g_{\varepsilon}$ are holomorphic functions in $\mathbb{D}$ and

$$
h_{\varepsilon}(z)=\frac{h(z)+\varepsilon g(z)}{1+\varepsilon g^{\prime}(0)}, \quad g_{\varepsilon}(z)=\frac{g(z)+\bar{\varepsilon} h(z)}{1+\overline{\varepsilon g^{\prime}(0)}} .
$$

We have to prove that $T_{\varphi}\left(f_{\varepsilon}\right) \in A(L)$ for any

$$
\varphi(z)=e^{i \theta} \frac{z+a}{1+\bar{a} z}, \quad a \in \mathbb{D}, \quad \theta \in \mathbb{R} .
$$

Let us fix $\varphi$. Denote $F_{\varepsilon}=T_{\varphi}\left(f_{\varepsilon}\right)=H_{\varepsilon}+\overline{G_{\varepsilon}}$, where

$$
\begin{aligned}
& H_{\varepsilon}^{\prime}(z)=\frac{h^{\prime}(\varphi(z))+\varepsilon g^{\prime}(\varphi(z))}{\left(h^{\prime}(\varphi(0))+\varepsilon g^{\prime}(\varphi(0))\right)(1+\bar{a} z)^{2}}, \\
& G_{\varepsilon}^{\prime}(z)=\frac{g^{\prime}(\varphi(z))+\bar{\varepsilon} h^{\prime}(\varphi(z))}{\overline{\left(h^{\prime}(\varphi(0))+\varepsilon g^{\prime}(\varphi(0))\right)}(1+\bar{a} z)^{2}} \cdot e^{2 i \theta} .
\end{aligned}
$$

Analogously, denote $T_{\phi}(f)=F=H+\bar{G} \in L$, where

$$
H^{\prime}(z)=\frac{h^{\prime}(\varphi(z))}{h^{\prime}(\varphi(0))(1+\bar{a} z)^{2}}, \quad G^{\prime}(z)=\frac{g^{\prime}(\varphi(z))}{\overline{h^{\prime}(\varphi(0))}(1+\bar{a} z)^{2}} \cdot e^{2 i \theta}
$$

and

$$
\varepsilon_{1}=\varepsilon \frac{h^{\prime}(\varphi(0))}{h^{\prime}(\varphi(0))} \cdot e^{2 i \theta} .
$$

We can write that $A_{\varepsilon}(F) \in A(L)$ because writing $A_{\varepsilon}(F)=\Phi_{\varepsilon}(z)=$ $\widehat{H}_{\varepsilon}+\overline{\widehat{G}}_{\varepsilon}$, where

$$
\widehat{H}_{\varepsilon}^{\prime}(z)=\frac{h^{\prime}(\varphi(z))+\varepsilon_{1} g^{\prime}(\varphi(z))}{\left(h^{\prime}(\varphi(0))+\varepsilon_{1} g^{\prime}(\varphi(0))\right)(1+\bar{a} z)^{2}}=H_{\varepsilon_{1}}^{\prime}(z)
$$

and

$$
\widehat{G}_{\varepsilon}^{\prime}(z)=\frac{g^{\prime}(\varphi(z))+\overline{\varepsilon_{1}} h^{\prime}(\varphi(z))}{\overline{\left(h^{\prime}(\varphi(0))+\varepsilon_{1} g^{\prime}(\varphi(0))\right)}(1+\bar{a} z)^{2}} \cdot e^{2 i \theta}=G_{\varepsilon_{1}}^{\prime}(z),
$$

we have $\Phi_{\varepsilon}(z)=F_{\varepsilon_{1}} \in A(L)$, for any $\varepsilon_{1},\left|\varepsilon_{1}\right|<1$, due to the fact that $\varepsilon$ was arbitrary and $|\varepsilon|<1$. 
Remark 1.1. As we see from the proof, the operators $T_{\varphi}$ and $A_{\varepsilon}$ do not commute, i.e. $A_{\varepsilon} \circ T_{\varphi} \neq T_{\varphi} \circ A_{\varepsilon}$. However, we have

$$
\begin{aligned}
\left\{A_{\varepsilon} \circ T_{\varphi}(f): f \in L,|\varepsilon|<1, \varphi \in \operatorname{Aut}(\mathbb{D})\right\} & \\
& =\left\{T_{\varphi} \circ A_{\varepsilon}(f): f \in L,|\varepsilon|<1, \varphi \in \operatorname{Aut}(\mathbb{D})\right\} .
\end{aligned}
$$

2. We start with a slight improvement of Theorem 3.3 from [5].

Theorem 2.1. If $L$ is $A L I F$, ord $L=\alpha, \alpha>0$ and $f \in L$, then

$$
\begin{aligned}
\left(1-\left|a_{-1}(f)\right|^{2}\right) & \frac{(1-r)^{2 \alpha-2}}{(1+r)^{2 \alpha+2}} \leq J_{f}(z) \\
& \leq \frac{(1+r)^{2 \alpha-2}}{(1-r)^{2 \alpha+2}}\left(1-\left|a_{-1}(f)\right|^{2}\right), \quad|z|=r<1 .
\end{aligned}
$$

The bounds in (2.1) are sharp and the sign of equality holds for the function

$$
f(z)=k_{\alpha}(z)+a_{-1} \overline{k_{\alpha}(z)}
$$

where

$$
k_{\alpha}(z)=\frac{1}{2 \alpha}\left[\left(\frac{1+z}{1-z}\right)^{\alpha}-1\right] .
$$

Observe that $f(z)$ is univalent for $\alpha \in(0,2]$, which follows from univalence of $k_{\alpha}(z)$ for these $\alpha$ and the invariance of univalent harmonic functions w.r.t. operator $A_{\varepsilon}$.

Proof. (Theorem 2.1) The proof is exactly the same as in [5], only the value $J_{f}(0)=1-\left|a_{-1}(f)\right|^{2}$ has to be taken into account. Namely, using the inequality from $[5]$ :

$$
\begin{aligned}
\frac{d}{d r} \log \left(\frac{1-r}{1+r}\right)^{2 \alpha} & \leq \frac{\partial}{\partial r}\left[\log \left(J_{f}\left(r e^{i \theta}\right) \cdot\left(1-r^{2}\right)^{2}\right)\right] \\
& \leq \frac{d}{d r} \log \left(\frac{1+r}{1-r}\right)^{2 \alpha}, \quad z=r e^{i \theta}
\end{aligned}
$$

after integration along the segment $[0, r], 0<r<1$, we obtain $(2.1)$.

For a linear invariant family $L$ of holomorphic functions the inverse theorem holds (see [4]), i.e. inequality (2.1) implies that ord $L \leq \alpha$.

The next theorem is in some sense inverse to Theorem 2.1. We do not assume even the linear invariance of the family $L$.

Theorem 2.2. Let $f \in L$ and assume that the upper bound in (2.1) holds for some $\alpha>0$. Then there exists $\varepsilon,|\varepsilon|<1$ such that

$$
\left|a_{2}(f)-\frac{\varepsilon}{2}\right| \leq \alpha \text {. }
$$

The inequality (2.3) is sharp, which means that in the right side of the inequality $|\varepsilon|<1$ we can not write any constant smaller than 1 . 
Proof. We will apply the same ideas from [3]. By the assumption, $f \in L$ satisfies (2.1) which implies that for $z=r e^{i \theta}$,

$$
\log J_{f}(z)-\log J_{f}(0) \leq(2 \alpha-2) \log (1+r)-(2 \alpha+2) \log (1-r) .
$$

For $r=0$ the above inequality after differentiation gives

$$
\left.\frac{1}{J_{f}(0)} \cdot \frac{\partial}{\partial r} J_{f}\left(r e^{i \theta}\right)\right|_{r=0} \leq 4 \alpha
$$

But

$$
\begin{aligned}
\frac{\partial}{\partial r} J_{f}(z)=\left[h^{\prime \prime}\left(r e^{i \theta}\right) \overline{h^{\prime}\left(r e^{i \theta}\right)}\right. & \left.e^{i \theta}-g^{\prime \prime}\left(r e^{i \theta}\right) \overline{g^{\prime}\left(r e^{i \theta}\right)} e^{i \theta}\right] \\
& +\left[\overline{h^{\prime \prime}\left(r e^{i \theta}\right) \overline{h^{\prime}\left(r e^{i \theta}\right)} e^{i \theta}-g^{\prime \prime}\left(r e^{i \theta}\right) \overline{g^{\prime}\left(r e^{i \theta}\right)} e^{i \theta}}\right] .
\end{aligned}
$$

Therefore, by (2.4) for every real $\theta$ we have

$$
\left.\frac{\partial}{\partial r}\left(J_{f}\left(r e^{i \theta}\right)\right)\right|_{r=0}=2 \operatorname{Re}\left\{e^{i \theta}\left(h^{\prime \prime}(0) \overline{h^{\prime}(0)}-g^{\prime \prime}(0) \overline{g^{\prime}(0)}\right)\right\} \leq 4 \alpha J_{f}(0) .
$$

Thus

$$
\left|h^{\prime \prime}(0)-\overline{g^{\prime}(0)} g^{\prime \prime}(0)\right| \leq 2 \alpha J_{f}(0),
$$

due to the fact that $h^{\prime}(0)=1$. The above inequality is equivalent to

$$
\frac{\left|a_{2}(f)-a_{-1}(f) \overline{a_{-2}(f)}\right|}{1-\left|a_{-1}(f)\right|^{2}} \leq \alpha .
$$

Let us put

$$
\omega(z)=\frac{g^{\prime}(z)}{h^{\prime}(z)}=\overline{a_{-1}}(f)+2\left(\overline{a_{-2}}(f)-a_{2}(f) \overline{a_{-1}}(f)\right) z+\ldots
$$

and

$$
\omega_{0}(z)=\frac{\omega(z)-\overline{a_{-1}}(f)}{1-a_{-1}(f) \omega(z)}=\frac{2\left(\overline{a_{-2}}(f)-a_{2}(f) \overline{a_{-1}}(f)\right)}{1-\left|a_{-1}(f)\right|^{2}} z+\ldots .
$$

Because $\omega_{0}(0)=0$ and $\left|\omega_{0}(z)\right|<1$ we have

$$
2\left|\frac{\overline{a_{-2}}(f)-a_{2}(f) \overline{a_{-1}}(f)}{1-\left|a_{-1}(f)\right|^{2}}\right| \leq 1 .
$$

On the other hand, (2.5) can be rewritten as

or

$$
\left|\frac{a_{2}(f)\left(1-\left|a_{-1}(f)\right|^{2}\right)+a_{-1}(f)\left(a_{2}(f) \overline{a_{-1}}(f)-\overline{a_{-2}}(f)\right)}{1-\left|a_{-1}(f)\right|^{2}}\right| \leq \alpha
$$

$$
\left|a_{2}(f)-\frac{\varepsilon}{2}\right| \leq \alpha \quad \text { where } \quad|\varepsilon|=\left|a_{-1}(f) 2 \frac{a_{2}(f) \overline{a_{-1}(f)}-\overline{a_{-2}(f)}}{1-\left|a_{-1}(f)\right|^{2}}\right|<1 .
$$


In order to prove the sharpness consider the function

$$
f_{0}(z)=h_{0}(z)+\overline{g_{0}(z)},
$$

where

$$
h_{0}(z)=k_{\alpha+\frac{1}{2}}(z), \quad g_{0}^{\prime}(z)=k_{\alpha+\frac{1}{2}}^{\prime}(z) \frac{z+x}{1+x z}, \quad x \in(0,1) .
$$

We have $a_{2}\left(f_{0}\right)=\alpha+\frac{1}{2}$ and

$$
\frac{J_{f_{0}}(z)}{J_{f_{0}}(0)}=\frac{\left|k_{\alpha+\frac{1}{2}}^{\prime}(z)\right|^{2}\left(1-\left|\frac{z+x}{1+x z}\right|^{2}\right)}{\left|k_{\alpha+\frac{1}{2}}^{\prime}(0)\right|^{2}\left(1-x^{2}\right)}=\left|\frac{(1+z)^{2\left(\alpha-\frac{1}{2}\right)}}{(1-z)^{2\left(\alpha+\frac{3}{2}\right)}}\right| \frac{1-|z|^{2}}{|1+x z|^{2}}
$$

Moreover,

$$
\begin{aligned}
\min _{|z|=r}\left(|1-z|^{2}|1+x z|^{2}\right) & =\min _{\theta \in \mathbb{R}}\left[\left(1-2 r \cos \theta+r^{2}\right)\left(1+2 x r \cos \theta+x^{2} r^{2}\right)\right] \\
& =(1-r)^{2}(1+x r)^{2},
\end{aligned}
$$

and the minimum is attained for $\theta=0$. Therefore,

$$
\max _{|z|=r} \frac{J_{f_{0}}(z)}{J_{f_{0}}(0)}=\frac{(1+r)^{2 \alpha-1}}{(1-r)^{2 \alpha+3}} \cdot \frac{(1-r)^{2}}{(1+x r)^{2}}<\frac{(1+r)^{2 \alpha-2+2 \delta(x)}}{(1-r)^{2 \alpha+2+\delta(x)}}
$$

which implies that inequality (2.3) for $f_{0}$ can be written in the form

$$
\left|\left(\alpha+\frac{1}{2}\right)-\frac{\varepsilon}{2}\right| \leq \alpha+\delta(x),
$$

where $0<\delta(x) \rightarrow 0$ if $x \rightarrow 1^{-}$, and

$$
\varepsilon=-a_{-1}\left(f_{0}\right) 2 \frac{a_{2}\left(f_{0}\right) \overline{a_{-1}\left(f_{0}\right)}-\overline{a_{-2}\left(f_{0}\right)}}{1-\left|a_{-1}\left(f_{0}\right)\right|^{2}}=x,
$$

because $2 \overline{a_{-2}\left(f_{0}\right)}=1-x^{2}+2 a_{2}\left(f_{0}\right) x$. This makes the result of Theorem 2.2 sharp.

Remark 2.1. Theorem 2.2 is also valid for holomorphic functions $f(z)$. In this case we have to put in the proof $\varepsilon=0$.

Remark 2.2. From the above proof we see that in the statement of Theorem 2.2 it is sufficient to assume that $f(z)$ satisfies only the right- (or left-) hand side of inequality (2.1).

Corollary 2.1. If the family $L$ is LIF and for any $f \in L$ inequality (2.1) holds, then ord $L \leq \alpha+\frac{1}{2}$. 
3. Now we introduce the definition of new order $\overline{\text { ord }} L$ (we will call the strong order) of a linear invariant family $(L I F)$ of harmonic mappings $L$. In terms of this new order one can formulate iff version of Theorem 2.1 without assuming family $L$ to be affine.

Definition 3.1. Let $L$ be $L I F$. The strong order $\overline{\text { ord }} L$ of a family $L$ of harmonic mappings $f$ is defined by the formula

$$
\overline{\operatorname{ord}} L=\sup _{f \in L} \frac{\left|a_{2}(f)-a_{-1}(f) \overline{a_{-2}}(f)\right|}{1-\left|a_{-1}(f)\right|^{2}} .
$$

Remark 3.1. In the case when $f$ is holomorphic in $\mathbb{D}$, definition (3.1) coincides with that introduced by Pommerenke in [4].

Definition 3.2. For any fixed $f \in L$ we define

$$
\overline{\operatorname{ord}} f=\sup _{\varphi \in \operatorname{Aut}(\mathbb{D})} \frac{\left|a_{2}\left(T_{\varphi}(f)\right)-a_{-1}\left(T_{\varphi}(f)\right) \overline{a_{-2}}\left(T_{\varphi}(f)\right)\right|}{1-\left|a_{-1}\left(T_{\varphi}(f)\right)\right|^{2}} .
$$

Of course, if $L$ is $L I F, \overline{\text { ord }} L=\alpha$, then $\overline{\text { ord }} f \leq \alpha$ for any $f \in L$.

Theorem 3.1. If $f \in L$, then $\overline{\operatorname{ord}} f \leq \alpha$ if and only if for every $F=F_{\psi}:=$ $T_{\psi}(f)$ and any $z \in \mathbb{D}$

$$
\frac{(1-r)^{2 \alpha-2}}{(1+r)^{2 \alpha+2}} \leq \frac{J_{F}(z)}{J_{F}(0)} \leq \frac{(1+r)^{2 \alpha-2}}{(1-r)^{2 \alpha+2}}, \quad|z|=r<1 .
$$

Proof. Assume first that $\overline{\operatorname{ord}} f \leq \alpha, \varphi(z)=\frac{z+a}{1+\bar{a} z}, z, a \in \mathbb{D}$ and $T_{\psi}(f)=$ $F=H+\bar{G}, \psi \in \operatorname{Aut}(\mathbb{D})$. Consider $F_{a}(z)=T_{\varphi}(F)=H_{a}+\bar{G}_{a}$, where $H, H_{a}, G$ and $G_{a}$ are holomorphic functions in $\mathbb{D}$. By direct calculations we find

$$
G_{a}^{\prime}(z)=\frac{G^{\prime}(\varphi(z))}{\overline{H^{\prime}(a)} \cdot(1+\bar{a} z)^{2}}, \quad H_{a}^{\prime}(z)=\frac{H^{\prime}(\varphi(z))}{H^{\prime}(a) \cdot(1+\bar{a} z)^{2}},
$$

and

$$
\begin{gathered}
G_{a}^{\prime}(0)=\frac{G^{\prime}(a)}{\overline{H^{\prime}(a)}}, \quad G_{a}^{\prime \prime}(0)=\frac{G^{\prime \prime}(a)\left(1-|a|^{2}\right)-2 \bar{a} G^{\prime}(a)}{\overline{H^{\prime}(a)}}, \\
H_{a}^{\prime \prime}(0)=\frac{H^{\prime \prime}(a)\left(1-|a|^{2}\right)}{H^{\prime}(a)}-2 \bar{a} .
\end{gathered}
$$

One can easily verify that

$$
J_{F_{a}}(z)=\frac{J_{F}(\varphi(z))}{\left|H^{\prime}(a)\right|^{2}|1+\bar{a} z|^{4}} .
$$

Moreover, for $z=r e^{i \theta} \in \mathbb{D}$ we have

$$
\left.\frac{\partial J_{F_{a}}\left(r e^{i \theta}\right)}{\partial r}\right|_{r=0}=2 \operatorname{Re}\left\{e^{i \theta}\left(H_{a}^{\prime \prime}(0)-G_{a}^{\prime \prime}(0) \overline{G_{a}^{\prime}(0)}\right)\right\},
$$


and by the definition of the order

$$
\begin{aligned}
\left.\frac{\partial J_{F_{a}}\left(r e^{i \theta}\right)}{\partial r}\right|_{r=0} & =\left.2 \operatorname{Re}\left\{e^{i \theta} \frac{\partial}{\partial z} J_{F_{a}}(z)\right\}\right|_{z=0} \\
& =2 \operatorname{Re}\left\{e^{i \theta}\left(2 a_{2}\left(F_{a}\right)-2 \overline{a_{-2}}\left(F_{a}\right) a_{-1}\left(F_{a}\right)\right\}\right. \\
& \leq 4 \alpha\left(1-\left|a_{-1}\left(F_{a}\right)\right|^{2}\right) .
\end{aligned}
$$

Therefore, from (3.4) we obtain

$$
\begin{aligned}
\left.\frac{\partial J_{F_{a}}\left(r e^{i \theta}\right)}{\partial r}\right|_{r=0}= & 2 \operatorname{Re}\left\{e ^ { i \theta } \left[\frac{H^{\prime \prime}(a)\left(1-|a|^{2}\right)}{H^{\prime}(a)}-2 \bar{a}\right.\right. \\
& \left.\left.-\frac{\overline{G^{\prime}(a)}}{\left|H^{\prime}(a)\right|^{2}}\left(G^{\prime \prime}(a)\left(1-|a|^{2}\right)-2 \bar{a} G^{\prime}(a)\right)\right]\right\} \\
= & 2 \operatorname{Re}\left\{e^{i \theta}\left[\frac{\left(1-|a|^{2}\right)}{\left|H^{\prime}(a)\right|^{2}} \cdot \frac{\partial J_{F}(a)}{\partial z}-2 \bar{a}\left(1-\left|\frac{G^{\prime}(a)}{H^{\prime}(a)}\right|^{2}\right)\right]\right\} .
\end{aligned}
$$

Choosing first $\theta=a, a \neq 0$, we obtain $\left(a=\varrho e^{i \theta}\right)$

$$
\left.\frac{\partial J_{F_{a}}\left(r e^{i \theta}\right)}{\partial r}\right|_{r=0}=\frac{1}{\left|H^{\prime}(a)\right|^{2}}\left[\left(1-|a|^{2}\right) \frac{\partial J_{F}\left(\varrho e^{i \theta}\right)}{\partial \varrho}-4|a| J_{F}\left(\varrho e^{i \theta}\right)\right] \text {. }
$$

Applying (3.5), we get

$$
\frac{1}{J_{F}\left(\varrho e^{i \theta}\right)} \frac{\partial J_{F}}{\partial \varrho}\left(\varrho e^{i \theta}\right) \leq \frac{4(\alpha+\varrho)}{1-\varrho^{2}} .
$$

Choosing now $\theta=\pi+a$, we obtain

$$
\frac{1}{J_{F}\left(\varrho e^{i \theta}\right)} \cdot \frac{\partial J_{F}\left(\varrho e^{i \theta}\right)}{\partial \varrho} \geq 4 \frac{\varrho-\alpha}{1-\varrho^{2}} .
$$

Writing together the above inequalities, we get

$$
\begin{aligned}
2 \frac{\partial}{\partial \varrho}\left(\log (1-\varrho)^{\alpha-1}-\log (1+\varrho)^{\alpha+1}\right) & \leq \frac{\partial\left(\log J_{F}\left(\varrho e^{i \theta}\right)\right)}{\partial \varrho} \\
& \leq 2 \frac{\partial}{\partial \varrho}\left(\log (1+\varrho)^{\alpha-1}-\log (1-\varrho)^{\alpha+1}\right) .
\end{aligned}
$$

Integration over the interval $[0, r]$ implies (3.3).

Assume now that the right-hand side of (3.3) holds for some $\alpha>0$ and $r \in(0,1)$. We have to prove now that $\overline{\operatorname{ord}} f \leq \alpha$.

Because for the function $F=T_{\varphi}(f)$ the inequality (3.3) holds, therefore we have

$$
\log J_{F}(z)-\log J_{F}(0) \leq(2 \alpha-2) \log (1+r)-(2 \alpha+2) \log (1-r) .
$$

This implies, as in the proof of Theorem 2.2, the validity of equality (2.5) for the function $F=T_{\varphi}(f)$, and we have $\overline{\operatorname{ord}} f \leq \alpha$. 
From the proof of the above theorem we obtain the following corollary.

Corollary 3.1. If $L$ is $L I F$, then $\overline{\text { ord }} L \leq \alpha$ if and only if the right-hand side of (3.3) holds for any $f \in L$ and for every $r \in(0, \delta), 0<\delta<1$.

If $L$ is $L I F$, then by Theorem 3.1 we can give an equivalent definition of the $\overline{\operatorname{ord}} L$. Namely,

\section{Corollary 3.2.}

$$
\begin{aligned}
\overline{\operatorname{ord}} L=\inf \left\{\alpha: \frac{J_{f}(z)}{J_{f}(0)} \leq\right. & \frac{(1+|z|)^{2 \alpha-2}}{(1-|z|)^{2 \alpha+2}} \\
& \text { for any } f \in L \text { and any } z \in \mathbb{D}\} .
\end{aligned}
$$

From Theorem 2.1 and Corollary 2.1 we derive the next corollaries.

Corollary 3.3. If $L$ is $L I F$, then

$$
\text { ord } L \leq \overline{\operatorname{ord}} L+\frac{1}{2} \text {. }
$$

Corollary 3.4. If $L$ is $L I F$, then

$$
\overline{\operatorname{ord}} L \leq \operatorname{ord} A(L), \quad \text { ord } A(L) \geq \operatorname{ord} L .
$$

If $L$ is $A L I F$, then

$$
\operatorname{ord} L-\frac{1}{2} \leq \overline{\operatorname{ord}} L \leq \text { ord } L .
$$

In particular, because the class $S_{H}$ of harmonic univalent functions in $\mathbb{D}$ is an $A L I F$, we have

$$
\operatorname{ord} S_{H}-\frac{1}{2} \leq \overline{\operatorname{ord}} S_{H} \leq \operatorname{ord} S_{H}
$$

Indeed, since $A(L)$ is a $L I F$, then by Theorem 2.1 for any $f \in A(L)$ inequality (3.3) holds with $\alpha=\operatorname{ord} A(L)$ and we have $\overline{\operatorname{ord}} L \leq \operatorname{ord} A(L)$. The second inequality ord $A(L) \geq$ ord $L$ follows by the relation $L \subset A(L)$.

From (3.7) and the fact that $\mathscr{L} A(L)=L$ if $L$ is an $A L I F$, we have (3.8).

Remark 3.2. The equality ord $L=\overline{\text { ord }} L$ is possible. Take for example $L=\mathscr{L} A\left(k_{\alpha}\right)$, where $k_{\alpha}$ is given by (2.2). However, the inequality ord $L \neq$ $\overline{\text { ord }} L$ can hold as well, as shows the following.

Example. Let $k_{\alpha}(z)$ be the generalized Koebe function given by (2.2).

Put $f_{\alpha}(z)=k_{\alpha}(z)+\overline{g(z)}$, where $g^{\prime}(z)=z k_{\alpha}^{\prime}(z)$. Consider the family $L=\mathscr{L}\left(f_{\alpha}\right)$, the linear- invariant hull of the function $f_{\alpha}$, i.e.

$$
\mathscr{L}\left(f_{\alpha}\right)=\left\{T_{\varphi}\left(f_{\alpha}\right): \varphi \in \text { Aut } \mathbb{D}\right\} .
$$

If $F \in \mathscr{L}\left(f_{\alpha}\right)$, then there exists $\varphi \in \operatorname{Aut}(\mathbb{D})$ such that

$$
F(z)=\frac{k_{\alpha}(\varphi(z))-k_{\alpha}(\varphi(0))}{k_{\alpha}^{\prime}(\varphi(0)) \varphi^{\prime}(0)}+\frac{\overline{g(\varphi(z))}-\overline{g(\varphi(0))}}{k_{\alpha}^{\prime}(\varphi(0)) \varphi^{\prime}(0)} .
$$


Denote ord $F=\operatorname{ord} \mathscr{L}(F)$. Therefore, ord $F=\operatorname{ord} k_{\alpha}=\alpha$ (see [4]) and ord $L=\alpha$.

Taking $\varphi(z)=\frac{z+a}{1+\bar{a} z},(a \in \mathbb{D})$ we find

$$
\begin{aligned}
\frac{J_{F}(z)}{J_{F}(0)} & =\left|\frac{\left(1+\frac{z+a}{1+\bar{a} z}\right)^{\alpha-1} \cdot(1-a)^{\alpha+1}}{\left(1-\frac{z+a}{1+\bar{a} z}\right)^{\alpha+1} \cdot(1+a)^{\alpha-1} \cdot(1+\bar{a} z)^{2}}\right|^{2} \frac{\left(1-\left|\frac{z+a}{1+\bar{a} z}\right|^{2}\right)}{1-|a|^{2}} \\
& =\left|\frac{\left(1+z \frac{1+\bar{a}}{1+a}\right)^{\alpha-1}}{\left(1-z \frac{1-\bar{a}}{1-a}\right)^{\alpha+1}}\right| \cdot \frac{1-|z|^{2}}{|1+\bar{a} z|^{2}} .
\end{aligned}
$$

For $z=r>0$ and $a \in(-1,0)$, when $a \rightarrow-1$ the right-hand side of the latter expression tends to $\frac{(1+r)^{2 \alpha-1}}{(1-r)^{2 \alpha+3}}$ and therefore, $\overline{\text { ord }} L \geq \alpha+\frac{1}{2}$ (in fact $\overline{\operatorname{ord}} L=\alpha+\frac{1}{2}$ ). So $\overline{\text { ord }} L \neq \operatorname{ord} L$.

Theorem 3.2. Assume that $f_{1}, f_{2} \in L$ and $L$ is LIF. If

$$
\frac{J_{f_{1}}(z)}{J_{f_{1}}(0)}=\frac{J_{f_{2}}(z)}{J_{f_{2}}(0)}
$$

for every $z \in \mathbb{D}$, then $\overline{\text { ord }} f_{1}=\overline{\text { ord }} f_{2}$.

Proof. We conclude the proof of Theorem 3.2 by Corollary 3.2 because

$\inf \left\{\alpha:(3.3)\right.$ holds for any $\left.F \in \mathscr{L}\left(f_{1}\right)\right\}$

$$
=\inf \left\{\alpha:(3.3) \text { holds for any } F \in \mathscr{L}\left(f_{2}\right)\right\},
$$

where $\mathscr{L}(f)$ is defined as above in the Example.

Definition 3.3. Put

$$
U_{\alpha}^{H}=\bigcup\{L: L \text { is } L I F \text { and } \overline{\text { ord }} L \leq \alpha\}
$$

and call it the universal LIF of strong order $\alpha$.

Remark 3.3. From the definition it is obvious that $f \in U_{\alpha}^{H}$ iff $\overline{\text { ord }} f \leq \alpha$.

Theorem 3.3. The family $U_{\alpha}^{H}$ is ALIF.

Proof. We have to prove that for any $f \in U_{\alpha}^{H}$ and $|\varepsilon|<1$, the function

$$
A_{\varepsilon}(f)=f_{\varepsilon}(z)=\frac{f(z)+\varepsilon \overline{f(z)}}{1+\varepsilon \overline{a_{-1}}(f)} \in U_{\alpha}^{H},
$$

i.e. $\overline{\text { ord }} f_{\varepsilon} \leq \alpha$. Putting

$$
\varepsilon=\varrho e^{i \beta}, \quad \varrho \in[0,1), \quad \beta \in \mathbb{R},
$$

we can find that

$$
\frac{J_{f_{\varepsilon}}(z)}{J_{f_{\varepsilon}}(a)}=\frac{J_{f}(z)}{J_{f}(a)}, \quad z, a \in \mathbb{D} .
$$


Indeed, we have for any harmonic function $f=u+i v$, where $u$ and $v$ are real functions: $J_{f}=u_{x} v_{y}-u_{y} v_{x}$. Therefore, for $f_{\varepsilon}=A_{\varepsilon}(f)$ we obtain

$$
\begin{aligned}
& J_{f_{\varepsilon}}(z)= \\
& =\frac{1}{\left|1+\varepsilon \overline{a_{-1}}(f)\right|^{2}}\left|\begin{array}{ll}
u_{x}(1+\varrho \cos \beta)+v_{x} \varrho \sin \beta & u_{y}(1+\varrho \cos \beta)+v_{y} \varrho \sin \beta \\
v_{x}(1-\varrho \cos \beta)+u_{x} \varrho \sin \beta & v_{y}(1-\varrho \cos \beta)+u_{y} \varrho \sin \beta
\end{array}\right| \\
& =\frac{\left(1-\varrho^{2} \cos ^{2} \beta\right) J_{f}(z)}{\left|1+\varepsilon \overline{a_{-1}}(f)\right|^{2}},
\end{aligned}
$$

which implies (3.10). By Corollary 3.2 putting $|z|=r$, we have

$\overline{\operatorname{ord}} f_{\varepsilon}=\inf \left\{\alpha: \frac{J_{F_{\varepsilon}}(z)}{J_{F_{\varepsilon}}(0)} \leq \frac{(1+r)^{2 \alpha-2}}{(1-r)^{2 \alpha+2}}\right.$ for any $\left.F_{\varepsilon}=T_{\varphi}\left(f_{\varepsilon}\right), \varphi \in \operatorname{Aut}(\mathbb{D})\right\}$.

But from (3.10)

$$
\frac{J_{F_{\varepsilon}}(z)}{J_{F_{\varepsilon}}(0)}=\frac{J_{f_{\varepsilon}}(\varphi(z))}{J_{f_{\varepsilon}}(\varphi(0))\left|1-\overline{\varphi^{-1}(0)} z\right|^{4}}=\frac{J_{F}(z)}{J_{F}(0)}, \quad F=T_{\varphi}(f) .
$$

Therefore,

$$
\begin{aligned}
\overline{\operatorname{ord}} f_{\varepsilon} & =\inf \left\{\alpha: \frac{J_{F}(z)}{J_{F}(0)} \leq \frac{(1+r)^{2 \alpha-2}}{(1-r)^{2 \alpha+2}} \text { for any } F=T_{\varphi}(f), \varphi \in \operatorname{Aut}(\mathbb{D})\right\} \\
& =\overline{\operatorname{ord}} f \leq \alpha,
\end{aligned}
$$

because $f \in U_{\alpha}^{H}$.

Corollary 3.5. If $L$ is $L I F$, then

$$
\overline{\operatorname{ord}} L \geq 1 \text {. }
$$

Proof. Assume on the contrary that $\overline{\operatorname{ord}} L<1$. Then from the left-hand side of (3.3) it follows that

$$
\frac{J_{F}(z)}{J_{F}(0)} \rightarrow+\infty, \quad \text { as } \quad|z|=r \rightarrow 1^{-} .
$$

This implies that the numerator of the above expression, which is $\left|h^{\prime}(z)\right|^{2}-$ $\left|g^{\prime}(z)\right|^{2} \rightarrow+\infty$, and therefore, $\left|h^{\prime}(z)\right| \rightarrow+\infty$. This is in contradiction with the minimum principle because we would have

$$
\min _{|z|=r<1}\left|h^{\prime}(z)\right| \leq 1,
$$

due to the fact that $h^{\prime}(0)=1$.

Acknowledgements. The authors would like to express their great thanks to the Referee, whose detailed and careful review helped to improve the presentation of the results.

The second author has been partially supported by the RFBR Grant No. 11-01-00952a. 


\section{REFERENCES}

[1] Bshouty, D., Hengartner, W., Univalent harmonic mappings in the plane, Ann. Univ. Mariae Curie-Skłodowska Sect. A 48 (1994), 13-42.

[2] Duren, P., Harmonic Mappings in the Plane, Cambridge University Press, Cambridge, 2004.

[3] Godula, J., Liczberski, P. and Starkov, V. V., Order of linearly invariant mappings in $C^{n}$, Complex Variables Theory Appl. 42 (2000), 89-96.

[4] Pommerenke, Ch., Linear-invariante Familien analytischer Funktionen I, Math. Annalen 155 (1964), 108-154.

[5] Schaubroeck, L. E., Subordination of planar harmonic functions, Complex Variables Theory Appl. 41, (2000), 163-178.

[6] Sheil-Small, T., Constants for planar harmonic mappings, J. London Math. Soc. 42 (1990), 237-248.

M. Sobczak-Kneć

Lublin University of Technology

ul. Nadbystrzycka 38D

20-618 Lublin

Poland

e-mail: m.sobczak-knec@pollub.pl
V. V. Starkov

Department of Mathematics

University of Petrozavodsk

ul. Lenina 33

185910 Petrozavodsk

Russia

e-mail: vstar@psu.karelia.ru

J. Szynal

Department of Mathematics

University of Economics and Innovations

ul. Mełgiewska 7-9

20-209 Lublin

Poland

e-mail: jan.szynal3@gmail.com

Received July 4, 2010 